\title{
Nonspecific protein binding can enhance the oscillatory regime of a biomolecular clock
}

\author{
Supravat Dey ${ }^{1}$, and Abhyudai Singh ${ }^{2}$
}

\begin{abstract}
Rhythms in gene regulatory networks are ubiquitous, from the circadian clock to the segmentation clock of vertebrates. There are many nonspecific protein binding sites (decoys) in a genome where regulatory proteins bind, and such decoys critically control the expression of a gene. The role decoys play on oscillatory regulatory networks is not well understood. Here, in the presence of decoy binding sites, we investigate the stability and the precision of the wellknown Goodwin oscillator, a minimal model for regulatory oscillators. We derive the stability criterion in the presence of decoys and find that increasing decoy abundance can expand the parameter space where oscillating solutions exist. If the Goodwin system does not show any oscillation without decoy binding sites, a sustained oscillation is possible in their presence. Finally, we study precision in oscillations using stochastic simulations and find that decoy sites can make the oscillation more precise in terms of reducing noise in both the time period and the amplitude. Whereas, in an open-loop circuit, the gene expression can become noisier in the presence of decoy sites.
\end{abstract}

\section{INTRODUCTION}

In living cells, the expression of a gene is inherently stochastic as biochemical reactions are occurring at very low copy numbers. For this reason, across a population of genetically identical cells under the same external environment, the mRNA and protein levels can show a great variability [1]-[5]. This variation is known as gene-expression noise. Depending on contexts, this noise in the gene-expression has many adverse or positive consequences [6]-[11]. For example, the gene expression noise can control cell fate [8], [12], can cause defects in developing embryos [13], [14]. The gene expression noise can enhance phenotypic diversity which is important for the survival of an organism in a population [9], [10].

Amid this randomness, many processes in life occur with great accuracies. A fascinating example is the existence of precise rhythms in biological process. The examples of biomolecular oscillators are ubiquitous, from the circadian clock to the segmentation clock of vertebrates [13]-[17]. Cells employ several regulatory mechanisms to create sustained oscillations [13], [18]. One of the simplest regulatory network that show sustained oscillations is the well-known Goodwin oscillator [19]. The essential ingredients that trigger oscillation in the Goodwin circuit are strong repression and "time delay" created by an intermediate protein. In this

\footnotetext{
1 Department of Electrical and Computer Engineering, University of Delaware, Newark, DE 19716, USA supravat.dey@gmail.com

${ }^{2}$ Department of Electrical and Computer Engineering, Department of Biomedical Engineering and Department of Mathematical Sciences, University of Delaware, Newark, DE 19716, USA absingh@udel. edu
}

article, we study effect of the nonspecific protein binding on the Goodwin oscillator.

There are many nonspecific (decoy) binding sites in a genome where regulatory proteins can bind with high affinities [20]. In specific binding, regulatory proteins bind to the specific site of a gene (known as promoter) and directly regulate its expression by activating or inhibiting the transcription process. The sequestration of these proteins to the decoy sites on the other hand modulates the activity of the regulatory protein and indirectly, controls the expression of the corresponding gene. The decoys has great therapeutic potential due to its ability to control the activity of the regulatory proteins [21]-[24]. Several recent studies investigate how the decoy binding affects the expression levels and the corresponding noises in various non-oscillatory circuits theoretically [25]-[30]. The decoys can affect the oscillatory dynamics as well. For an NF-kB circuit it has been shown that decoy reduces the oscillation amplitude [31]. For activator-repressor motifs, the versatile role of decoys for tuning amplitude and frequency of the oscillation has been studied [32]. However a general understanding of decoys role on the mean and the noisy oscillatory dynamics is still missing.

Here, we investigate the role of decoys on the Goodwin oscillator by formulating a model where decoys bind to the inhibitory proteins and control its activity. In this study, we consider that both the free and bound proteins can decay and assume the decay rates to be the same. This decay could be due to active degradation process such as ubiquitin-mediated proteolysis or because of dilution due to cell growth/division [26], [33]. We note that previous studies often assume the decoy binding protects proteins from the degradations [27] [30]. Before investigating the oscillatory circuit, we first discuss the role of the decoy on the activity of a protein in an open-loop circuit. We obtain the analytical expression for the free protein noise, using the linear noise approximation [34]. We find that the noise in free protein shows an enhancement for limited decoy abundances. Then, we derive the stability condition for the Goodwin system in the presence of decoy binding sites and find that decoy abundance increases the regime in the parameter space where oscillating solutions exist. Finally, we solve the stochastic dynamics using Monte Carlo simulations [35] to compute the noise in the time period and amplitude of the oscillations, as an analytical solution for the noise in the oscillatory system is hard. We find that decoys buffer the noise in the Goodwin oscillator, as opposed to the open-loop circuit. 


\section{Bursty protein synthesis \\ from a constitutive gene}

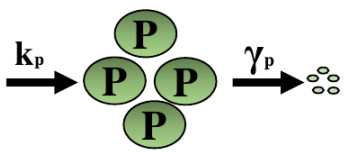

Interactions with decoy binding sites

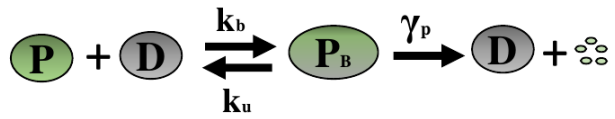

Fig. 1. Biochemical reactions for studying the noise properties in the presence of decoys in the open-loop circuit. The production of protein occurs in bursts with a frequency $k_{p}$ and an average burst size $\left\langle B_{p}\right\rangle$. The proteins reversibly bind to decoy binding sites with fast rates and produce the bound complex. The protein can degrade with the same degradation rate, $\gamma_{p}$, irrespective of it is in the free or bound state.

\section{AN OPEN-LOOP CIRCUIT IN THE PRESENCE OF DECOY BINDING SITES}

Here, we study how decoy sites affect the noise in protein counts in the open loop circuit shown in Fig. 1. We consider a bursty production of the protein $P$ from a constitutive gene. The frequency of the burst is $k_{p}$ and the burst sizes are drawn from a geometrical distribution, $\alpha_{p}(i)$ [36]-[39]. We choose the following shifted geometric distribution [28],

$$
\alpha_{p}(i)=\left(1-1 /\left\langle B_{p}\right\rangle\right)^{i-1} /\left\langle B_{p}\right\rangle \text { for } i \in\{1,2,3, \ldots\} \text {. }
$$

The free proteins bind to decoy binding sites reversibly and form bound proteins $P_{b}$. We assume the degradation rates for both free and bound proteins are the same, denoted as $\gamma_{p}$. The total number of decoy binding sites $N$ is assumed to be fixed. The binding and unbinding rates, $k_{b}$ and $k_{u}$, are very large compared to the production and degradation rates.

Symbols and notations used: The uppercase symbols represent the species name. The bold lowercase symbols denote the stochastic variables. The $\langle\cdot\rangle$, and $\overline{\langle\cdot\rangle}$ denote the transient and steady-state averages. For examples, $\boldsymbol{p}$ is the stochastic variables representing copy numbers of the protein $P$, and $\overline{\langle\boldsymbol{p}\rangle}$ is its steady-state mean. The normal (not bold) lowercase symbols such as $p$ is the deterministic variables for $P$ and $\tilde{p}$ is the corresponding fixed point value which can be obtained by setting time the derivatives terms of the dynamical equations to zero.

Given the copy numbers of the free and bound proteins at time $t, \boldsymbol{p}$ and $\boldsymbol{p}_{b}$, the probabilities of the production, binding, unbinding and degradations of proteins, occurring in the next infinitesimal time $(t, t+d t)$ are given by

$$
\begin{aligned}
& \mathbb{P}\left(\boldsymbol{p}+i, \boldsymbol{p}_{b}\right)=k_{p} \alpha_{p}(i) d t, \\
& \mathbb{P}\left(\boldsymbol{p}-1, \boldsymbol{p}_{b}+1\right)=k_{b} \boldsymbol{p}\left(N-\boldsymbol{p}_{b}\right), \\
& \mathbb{P}\left(\boldsymbol{p}+1, \boldsymbol{p}_{b}-1\right)=k_{u} \boldsymbol{p}_{b}, \\
& \mathbb{P}\left(\boldsymbol{p}-1, \boldsymbol{p}_{b}\right)=\gamma_{p} \boldsymbol{p} d t, \\
& \mathbb{P}\left(\boldsymbol{p}, \boldsymbol{p}_{b}-1\right)=\gamma_{p} \boldsymbol{p}_{b} d t .
\end{aligned}
$$

For the above system, we can write the Chemical Master Equation (CME) that provides the time evolution of the joint probability density function $q\left(\boldsymbol{p}, \boldsymbol{p}_{b}, t\right)$, for observing $\boldsymbol{p}$ free protein count, and $\boldsymbol{p}_{b}$ bound protein count within time $t$ and $t+d t[40],[41]$

$$
\begin{aligned}
& \frac{d q\left(\boldsymbol{p}, \boldsymbol{p}_{b}, t\right)}{d t}=k_{p} \sum_{i=1}^{\boldsymbol{p}} \alpha_{p}(i) q\left(\boldsymbol{p}-i, \boldsymbol{p}_{b}, t\right) \\
& +\gamma_{p}(\boldsymbol{p}+1) p\left(\boldsymbol{p}+1, \boldsymbol{p}_{b}, t\right)+\gamma_{p}\left(\boldsymbol{p}_{b}+1\right) q\left(\boldsymbol{p}, \boldsymbol{p}_{b}+1, t\right) \\
& +k_{b}(\boldsymbol{p}+1)\left(N-\boldsymbol{p}_{b}+1\right) q\left(\boldsymbol{p}+1, \boldsymbol{p}_{b}-1, t\right) \\
& +k_{u}\left(\boldsymbol{p}_{b}+1\right) q\left(\boldsymbol{p}-1, \boldsymbol{p}_{b}+1, t\right)-k_{p} q\left(\boldsymbol{p}, \boldsymbol{p}_{b}, t\right) \\
& -\left[\gamma_{p}\left(\boldsymbol{p}+\boldsymbol{p}_{b}\right)+k_{u} \boldsymbol{p}_{b}+k_{b} \boldsymbol{p}\left(N-\boldsymbol{p}_{b}\right)\right] q\left(\boldsymbol{p}, \boldsymbol{p}_{b}, t\right) .
\end{aligned}
$$

As the terms involving bindings are nonlinear, the exact solution for the full probability density $q\left(\boldsymbol{p}, \boldsymbol{p}_{b}, t\right)$ is hard. In fact, we will see, the exact solution of moment dynamics for the above equation is also impossible. We use the wellknown Linear Noise Approximation [34], [42]-[45] to obtain a closed-form formula the noise.

\section{A. The deterministic dynamics}

The deterministic dynamics free and bound protein level for the open-loop circuit are given by the chemical rate equations,

$$
\begin{aligned}
\frac{d p}{d t} & =k_{p}\left\langle B_{p}\right\rangle-\gamma_{p} p-k_{b}\left(N-p_{b}\right) p+k_{u} p_{b}, \\
\frac{d p_{b}}{d t} & =-\gamma_{p} p+k_{b}\left(N-p_{b}\right) p-k_{u} p_{b},
\end{aligned}
$$

where $\left\langle B_{p}\right\rangle$ is the average burst size for the protein. The equilibrium solution of free protein count can be obtained by setting time derivatives of Eq. (4) to zero. This system of second-order nonlinearity has a unique physical solution. The other solution is unphysical, as it yields negative concentrations for free and bound protein for positive rate constants. In the limit of fast binding/unbinding, the equilibrium value of free protein concentration is given by

$\tilde{p}=\frac{1}{2}\left[\tilde{p}_{0}-\left(k_{d}+N\right)+\sqrt{\left(\tilde{p}_{0}-\left(k_{d}+N\right)\right)^{2}+4 k_{d} \tilde{p}_{0}}\right]$,

where $\tilde{p}_{0}=k_{p}\left\langle B_{p}\right\rangle / \gamma_{p}$ is the fixed point solution without decoy binding sites, and $k_{d}=k_{u} / k_{b}$ is the dissociation constant. The fixed point value of bound proteins in terms of free protein level is given by,

$$
\tilde{p}_{b}=N \tilde{p} /\left(k_{d}+\tilde{p}\right) .
$$

Note that the free protein counts $\tilde{p}$ decreases to zero as we increase the total number of decoy binding sites $N$ [25]. Whereas, if bound proteins are protected from the 


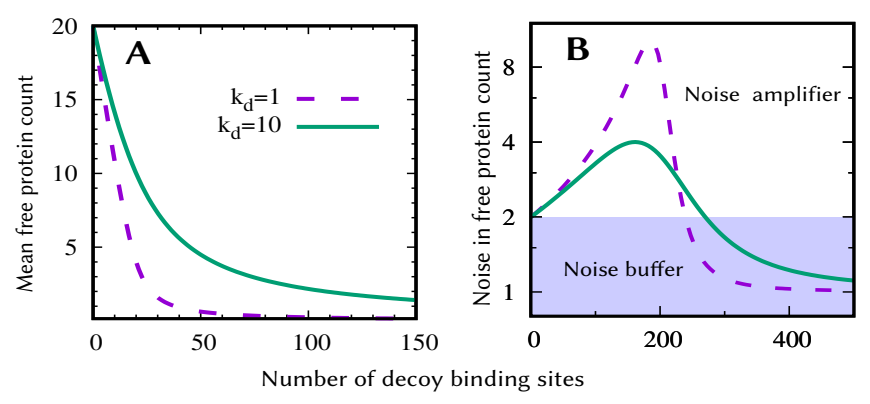

Fig. 2. The decoy binding sites can enhances the noise in the free protein counts. (A) The steady-sate mean protein count decays monotonically as function of $N$. The decay becomes faster for a smaller dissociation constant $k_{d}$. (B) The steady-state Fano fators in free protein count show a nonmonotonic behavior with $N$. For intermediate values of $N$, there is an enhancement in the noise. For large values of $N$, the noise approaches to the Poissonian limit. The noise enhancement becomes stronger for a smaller $k_{d}$. Parameters used: $\left\langle B_{p}\right\rangle=2, k_{p}=1$, and $\gamma_{p}=0.1$.

degradation $\tilde{p}=\tilde{p}_{0}$ independent of $N$ [26], [28]. Fig. 2(A) shows how the mean free protein level decreases with $N$. The decay becomes stiffer for larger binding affinities (smaller dissociation constants).

\section{B. Noise in free protein counts}

To calculate the noise in free protein counts, we need to compute all the statistical moments up to the second order. From the CME for our model (Eq. 3), we obtain the dynamics of any arbitrary moment $\left\langle\boldsymbol{p}^{m_{1}} \boldsymbol{p}_{b}^{m 2}\right\rangle$ (for $m_{1}, m_{2} \in$ $0,1,2, .$.

$$
\begin{gathered}
\frac{d\left\langle\boldsymbol{p}^{m_{1}} \boldsymbol{p}_{b}^{m_{2}}\right\rangle}{d t}=\left\langle k_{p} \sum_{i=1}^{\infty} \alpha_{p}(i)\left[(\boldsymbol{p}+i)^{m_{1}}-\boldsymbol{p}^{m_{1}}\right] \boldsymbol{p}_{b}^{m_{2}}\right\rangle \\
+\left\langle k_{b} \boldsymbol{p}\left(N-\boldsymbol{p}_{b}\right)\left[(\boldsymbol{p}-1)^{m_{1}}\left(\boldsymbol{p}_{b}+1\right)^{m_{2}}-\boldsymbol{p}^{m_{1}} \boldsymbol{p}_{b}^{m_{2}}\right]\right\rangle \\
+\left\langle k_{u} \boldsymbol{p}_{b}\left[\left(\boldsymbol{p}_{b}+1\right)^{m_{1}}\left(\boldsymbol{p}_{b}-1\right)^{m_{2}}-\boldsymbol{p}^{m_{1}} \boldsymbol{p}_{b}^{m_{2}}\right]\right\rangle \\
+\left\langle\gamma_{p} \boldsymbol{p}\left[(\boldsymbol{p}-1)^{m_{1}}-\boldsymbol{p}^{m_{1}}\right] \boldsymbol{p}_{b}^{m_{2}}\right\rangle \\
+\left\langle\gamma_{p} \boldsymbol{p}_{b}\left[\left(\boldsymbol{p}_{b}-1\right)^{m_{2}}-\boldsymbol{p}_{b}^{m 2}\right] \boldsymbol{p}^{m_{1}}\right\rangle .
\end{gathered}
$$

The exact analytical solution for moments do not exist as the nonlinearity of binding reaction makes the moments dynamics unclosed [46]-[52], i.e., the dynamical equation of a lower order moment depends on the higher order moments. We do the linear noise approximation [34] by linearizing the nonlinear term $k_{b} \boldsymbol{p} \boldsymbol{p}_{b}$ around the mean for free and bound protein count $\langle\boldsymbol{p}\rangle$ and $\left\langle\boldsymbol{p}_{b}\right\rangle$ as $k_{b} \boldsymbol{p} \boldsymbol{p}_{b}=$ $k_{b}\left(\boldsymbol{p}\left\langle\boldsymbol{p}_{b}\right\rangle+\langle\boldsymbol{p}\rangle \boldsymbol{p}_{b}-\langle\boldsymbol{p}\rangle\left\langle\boldsymbol{p}_{b}\right\rangle\right)$ [28]. In this approximation limit, the first order moment dynamics reduces to the deterministic dynamics (Eq. (4)) and $\langle\boldsymbol{p}\rangle=p$, and $\left\langle\boldsymbol{p}_{b}\right\rangle=p_{b}$. By solving the second order linearized dynamical equations at the steady state, we obtain the noise formula. We quantify the noise in free protein count by the Fano factor $F_{p}$, the ratio of the variance to the mean. In the limit of fast binding/unbinding, the analytical expression is given by

$$
\begin{aligned}
F_{p} & =\left(\overline{\left\langle\boldsymbol{p}^{2}\right\rangle}-\overline{\langle\boldsymbol{p}\rangle}^{2}\right) / \overline{\langle\boldsymbol{p}\rangle} \\
& =1+\frac{\left[\left(\left\langle B_{p}\right\rangle-1\right) \overline{\langle\boldsymbol{p}\rangle_{0}}+f^{2} N\right] \overline{\langle\boldsymbol{p}\rangle}}{\left(\overline{\langle\boldsymbol{p}\rangle_{0}}-f^{2} N\right)(N f(1-f)+\overline{\langle\boldsymbol{p}\rangle})},
\end{aligned}
$$

where the steady-state averages are denoted using the notation $\overline{\langle\cdot\rangle}, f=\overline{\left\langle\boldsymbol{p}_{b}\right\rangle} / N$ is the fraction of the bound decoys, ${\overline{\langle\boldsymbol{p}}\rangle_{0}}=\tilde{p}_{0}=k_{p}\left\langle B_{p}\right\rangle / \gamma_{p}$ is the steady-state value in the absence of decoy sites, and $\overline{\langle\boldsymbol{p}\rangle}=\tilde{p}$ and $\overline{\left\langle\boldsymbol{p}_{b}\right\rangle}=\tilde{p_{b}}$ are given by Eqs. 5 and 6 . In the absence of any decoy sites, the exact analytical expression noise can be obtained [53]. In this limit, the noise becomes $F_{p}=F_{p, 0}=\left\langle B_{p}\right\rangle$. In the limit of large decoy abundance, it can be shown that the noise approaches to the Poisssonian limit, i.e. $F_{p} \rightarrow 1$ as $N \rightarrow \infty$.

In Fig. 2(B), we plot the Fano factor in the free protein counts. The noise in protein count shows a nonmonotonic behavior. As a function of $N$, the noise increases to a maximum value and then decays to the Poissonian limit $\left(F_{p}=1\right)$. We note this noise enhancement is absent in the case when proteins bound to decoys are protected from the degradation [26], [28]. In the latter case, the noise monotonically decays to the Poissonian limit [28].

\section{The Goodwin OsCILlator IN PRESENCE OF DECOY BINDING SITES}

How does the nonspecific protein binding affect the precision of biological clocks? To address this question, we study the effect of the decoy bindings on a simple oscillatory system, namely the Goodwin oscillator [19]. Therefore, we first introduce the oscillator and do basic stability analysis. Then, we perform stability in the presence of decoy binding sites. Finally, we investigate the precision in the oscillations using stochastic simulations.

\section{A. The stability of the Goodwin oscillator}

The Goodwin oscillator is one of the minimal regulatory circuits that generates sustained oscillations. The ingredients that trigger oscillation are strong repression and "time delay" created by an intermediate protein. The biochemical reactions in the stochastic model of Goodwin oscillator are shown in Fig. 3. The deterministic dynamics of Goodwin oscillator is given by the chemical rate equations

$$
\begin{aligned}
& \frac{d m}{d t}=\frac{k_{m}}{1+\left(p / p_{c r i t}\right)^{h}}-\gamma_{m} m, \\
& \frac{d e}{d t}=k_{e} m-\gamma_{e} e, \\
& \frac{d p}{d t}=k_{p}^{0}\left\langle B_{p}\right\rangle e-\gamma_{p} p .
\end{aligned}
$$

The mRNA $M$ translated into protein $E$ and this protein activates the production of protein $P$. Finally, the protein $P$ represses the mRNA production and the Hill function for the repression is $f(p)=1 /\left[1+\left(p / p_{\text {crit }}\right)^{h}\right]$. Here, $h$ is the Hill coefficient and $p_{\text {crit }}$ is the repression coefficient, the level of $p$ at which half-maximal repression is achieved. The productions rates are $k_{m}, k_{e}$, and $k_{p}^{0}$, and the degradation 
Biochemical reactions in Goodwin Oscillator

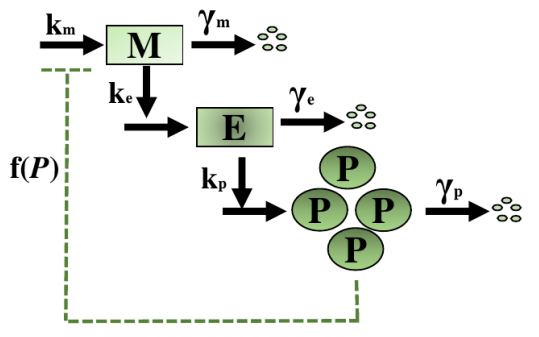

Interactions with decoy binding sites

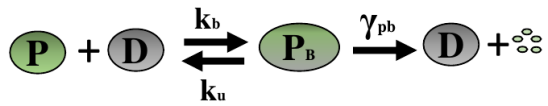

Fig. 3. Biochemical reactions in Goodwin oscillator in the presence of decoy binding sites. The regulatory protein $P$ inhibits the transcription of mRNA $M$. The mRNA translated into the protein $E$. The protein $E$ activates the bursty production of $P$. In the presence of $D$, the inhibitory protein reversibly binds to them with faster rates and produces bound complex $P_{b}$. The protein $P$ can degrade, irrespective of it is in the free or bound state.

rates are $\gamma_{m}, \gamma_{e}$, and $\gamma_{p}$. We consider the bursty production for protein $P$ with a burst size distribution given by Eq. (1). Note that a rescale of the production rate $k_{p}^{0}$ by its average burst size leads to the same deterministic dynamics as in the case of a simple birth process where the increment of protein counts happens by 1 at each time of production. However, it will be interesting to see how the precision in the oscillating pattern depends on $\left\langle B_{p}\right\rangle$.

Although the Goodwin system is highly nonlinear, it can be shown that there is a unique equilibrium point. This uniqueness comes from the negative regulation. Whereas a positive regulation can lead to multi-stability and this is absent in the Goodwin oscillator. Let $\left(\tilde{m}_{0}, \tilde{e}_{0}, \tilde{p}_{0}\right)$ be the fixed point. The Jacobian matrix at the equilibrium point is

$$
\mathbf{A}=\left(\begin{array}{ccc}
-\gamma_{m} & 0 & -g \\
k_{e} & -\gamma_{e} & 0 \\
0 & k_{p} & -\gamma_{p}
\end{array}\right)
$$

where

$$
g=\frac{h \gamma_{m} \gamma_{e} \gamma_{p}\left(1-f\left(\tilde{p}_{0}\right)\right)}{k_{e} k_{p}^{0}} .
$$

The characteristic polynomial of the Jacobian has the form $Q(x)=x^{3}+a x^{2}+b x+c$. The criteria for stable polynomial are: (i) $a, b, c>0$, and (ii) $a b>c$ [54]. The first condition always holds true. The second condition implies that for an unstable (oscillatory) solution, the value of the Hill coefficient must be greater than a threshold value $h_{0}^{t h r}$ :

$$
h>h_{0}^{t h r}:=\frac{\left(\gamma_{m}+\gamma_{e}\right)\left(\gamma_{e}+\gamma_{p}\right)\left(\gamma_{p}+\gamma_{m}\right)}{\gamma_{m} \gamma_{e} \gamma_{p}\left(1-f\left(\tilde{p}_{0}\right)\right)} .
$$

The functional form of the $h_{0}^{t h r}$ suggests that its minimum value is $8 /\left(1-f\left(\tilde{p}_{0}\right)\right)>8$. Therefore, to obtain an oscillatory
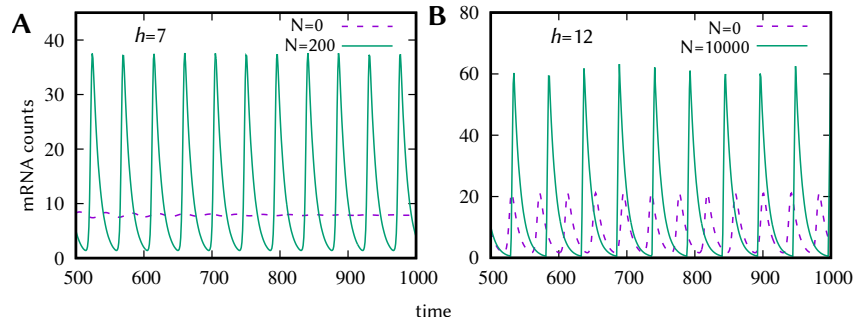

Fig. 4. The presence of decoys triggers oscillation in the mRNA counts: (A) For $h=7$ : In the absence of decoy, there is no oscillation, but the decoy's presence induces oscillation. (B): For $h=12$ : The oscillation is observed in the presence or absence of decoys. Interestingly, the decoy abundances increases the time period and the amplitude of the oscillation. Parameters used: $\left\langle B_{p}\right\rangle=1, k_{m}=20, k_{e}=1, k_{p}(0)=0.2$, $k_{d}=1, \gamma_{m}=\gamma_{e}=\gamma_{p}=0.1$, and $p_{\text {crit }}=100$.

solution the value of the Hill coefficient must be greater than 8 [55].

\section{B. The stability in the presence of decoy binding sites}

Here, we consider binding of inhibitory proteins to the decoy sites (see Fig. 3). Like the study of the open-loop circuit, we assume the total decoy binding sites $N$ is constant, and proteins decay with the same rate $\gamma_{p}$, regardless of binding to the decoys. The dynamics of protein and bound proteins are given by

$$
\begin{aligned}
& \frac{d p}{d t}=k_{p}\left\langle B_{p}\right\rangle e-k_{b}\left(N-p_{b}\right) p+k_{u} p_{b}-\gamma_{p} p, \\
& \frac{d p_{b}}{d t}=k_{b}\left(N-p_{b}\right) p-k_{u} p_{b}-\gamma_{p} p_{b} .
\end{aligned}
$$

The presence of the decoy does not introduce any multistability, as we have seen in the case of the open-loop circuit. However, it alters the equilibrium values of $m, p$, and $e$ from $\tilde{m}_{0}, \tilde{e}_{0}$, and $\tilde{p}_{0}$. In this study, we fix the equilibrium values to the corresponding values when there is no decoys, i.e. $\tilde{m}=\tilde{m}_{0}, \tilde{e}=\tilde{e}_{0}$, and $\tilde{p}=\tilde{p}_{0}$. In this way, we can compare the stability in the presence and absence of decoys around the "same" equilibrium point. To do that we increase the synthesis rate $k_{p}$ with $N$ in the following way,

$$
k_{p}(N):=k_{p}=k_{p}^{0}\left(1+\frac{N k_{b}}{\gamma_{p}+k_{b}\left(k_{d}+\tilde{p}_{0}\right)}\right) .
$$

The four dimensional Jacobian matrix at the equilibrium point is

$\mathbf{A}=\left(\begin{array}{cccc}-\gamma_{m} & 0 & -g & 0 \\ k_{e} & -\gamma_{e} & 0 & 0 \\ 0 & k_{p}(N) & -\gamma_{p}-l & k_{b}\left(k_{d}+\tilde{p}_{0}\right) \\ 0 & 0 & l & -\gamma_{p}-k_{b}\left(k_{d}+\tilde{p}_{0}\right)\end{array}\right)$

where

$$
l=\frac{k_{b} N\left(\gamma_{p}+k_{b} k_{d}\right)}{\gamma_{p}+k_{b}\left(k_{d}+\tilde{p}_{0}\right)}
$$

The characteristic polynomial of the Jacobian has the form $Q(x)=x^{4}+a x^{3}+b x^{2}+c x+d$. The criteria for stable 

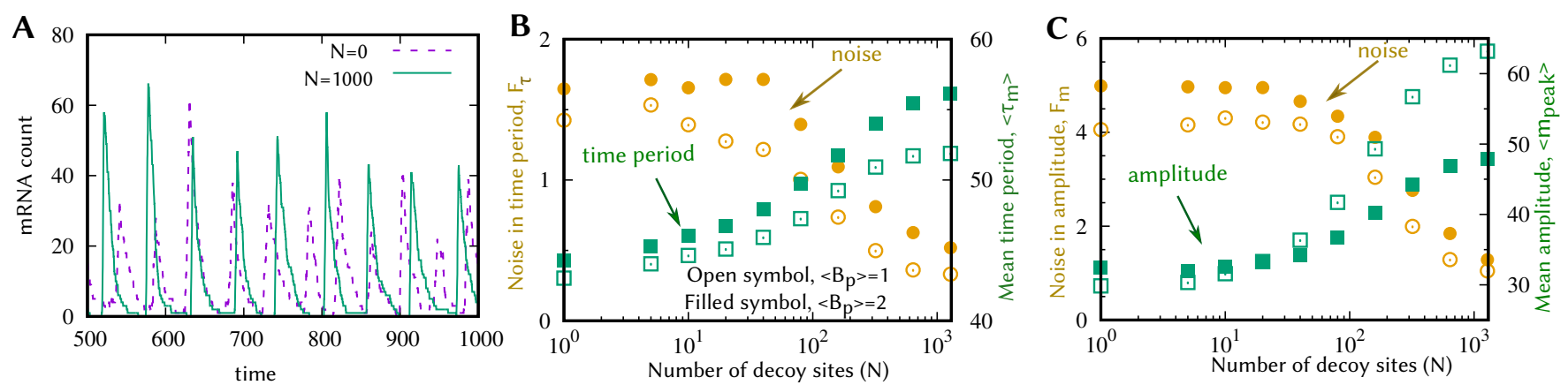

Fig. 5. The stochastic trajectories, the mean and the noise behavior in the presence of decoy sites for two different burst sizes: (A) The stochastic trajectories shows in the presence of decoy sites the amplitude and time period is larger than that of without decoys $\left(\left\langle B_{p}\right\rangle=2\right)$. (B) The average time period (squares) and its noise (circles) as a function of $N$ : The average time period increases, while the noise decrease with $N$. (C) The average amplitude (squares) and its noise (circles) as a function of $N$ : Similar to the time period, the average amplitude increases and the noise decrease with $N$. Parameters used: $k_{m}=20, k_{e}=1 k_{d}=1, \gamma_{m}=\gamma_{e}=\gamma_{p}=0.1, h=12$, and $p_{\text {crit }}=100 . k_{p}^{0}=0.2$ for $\left\langle B_{p}\right\rangle=1$ and $k_{p}^{0}=0.1$ for $\left\langle B_{p}\right\rangle=2$.

polynomial are: (i) $a, b, c, d>0$, (ii) $a b>c$, and (iii) $a b c-c^{2}>a^{2} d$ [54]. As the rate constants are positive, the first condition is always true. In the limit of fast/binding unbinding, considering the highest order term in $k_{b}$, one can show that the second condition will also hold true for any physical parameters. The third condition gives the condition for the stability in this system. Again considering the highest order term in $k_{b}$ one can show unstable solution exist if

$$
h>h_{0}^{t h r}\left(1-\frac{k_{d} N}{\left(k_{d}+\tilde{p}_{0}\right)\left(k_{d}+\tilde{p}_{0}+N\right)}\right) .
$$

The above equation suggests that one can obtain oscillation even for $h<8$ in the presence of decoys. Therefore, the decoy can enhance the region of instability. For $h>h_{0}^{t h r}$, the Eq. 16 holds true for any $N$, and hence the decoy abundance cannot kill the oscillation that was present without decoys binding sites.

We solve the deterministic dynamics given by the ordinary differential equations (Eqns. 9 (a), (b), and 13), numerically. The time series data of mRNA level are presented in the Fig. 4 for $h=7$ and $h=12$. For $h=7$, there is no sustained oscillation in the mRNA count when there is no decoys. Interestingly, in the presence of decoy, a sustained oscillation can be seen even for $h=7$. For $h=12$, sustained oscillations are observed both in the presence and absence of the decoy sites. However, the decoy abundance increases the time period and the amplitude of the oscillations. For an NF-kB circuit it has been shown that decoy reduces the oscillation amplitude [31]. For activator-repressor motifs, it is known that the decoy binding sites can tune the amplitude and the frequency, and can induce or kill the oscillations depending on whether decoys binds to the activators or repressors [32].

\section{Noise properties using stochastic simulations}

Here, we perform stochastic simulations to compute the precision in the time period and amplitude (the peak height). For exact stochastic simulations, we use kinetic Monte Carlo method due to Gillespie [35]. The typical time trajectories for the mRNA are presented in Fig. 5. We quantify the noise in time period of the oscillation in mRNA count using the Fano factor,

$$
F_{\tau}=\frac{\left\langle\tau_{m}^{2}\right\rangle-\left\langle\tau_{m}\right\rangle^{2}}{\left\langle\tau_{m}\right\rangle}
$$

where, $\left\langle\tau_{m}\right\rangle$ is the mean time period, and the second moment $\left\langle\tau_{m}^{2}\right\rangle$ at the oscillatory steady state. We also quantify the noise in the amplitude. For this, we compute the mRNA counts at peaks and define the following Fano factor

$$
F_{m}=\frac{\left\langle\boldsymbol{m}_{\text {peak }}^{2}\right\rangle-\left\langle\boldsymbol{m}_{\text {peak }}\right\rangle^{2}}{\left\langle\boldsymbol{m}_{\text {peak }}\right\rangle}
$$

where $\left\langle\boldsymbol{m}_{\text {peak }}\right\rangle$ and $\left\langle\boldsymbol{m}_{\text {peak }}^{2}\right\rangle$ is the first and second moment of the mRNA counts at the peaks at the oscillatory steady state.

In Fig. 5(B) and (C), we plot the mean and the noise behavior in the time period and amplitude of mRNA count for both the cases. The amplitude and time period increase with $N$ as observed in the deterministic case. We observe that the noise in the amplitude is quite large compared to that of time period. The increase in the burst size can make the system more noisy, as expected. The both noises in the time period and amplitude decrease as a function of $N$. In contrast, decoy can enhance in the open-loop circuit (see Fig. 2).

\section{Summary AND Discussion}

In summary, we have investigated the effect of nonspecific protein binding in the noise in gene expression for an open loop-circuit and an oscillatory regulatory circuit, in the limit of fast binding/unbinding. For the open-loop circuit, we have obtained an analytical expression for the noise in free protein counts considering proteins that are bound to the decoys are subjected to degrade, using the linear noise approximation. The case when bound proteins are stable were studied earlier in [26], [28]. We have found that, depending on the number 
of binding sites and binding affinity to the proteins, decoys can act as noise amplifier, whereas, decoys always suppress noise when binding protects proteins from the degradation [26], [28].

For the oscillatory regulatory circuit, we have studied the stability and the precision in the well-known Goodwin oscillator [19] in the presence of decoy binding sites, by incorporating decoy binding with the inhibitory proteins. By keeping the equilibrium values the same with the no decoy case, we have derived the condition for oscillatory solutions. We have found that decoy binding increases the parameter regime for the existence of oscillatory solutions. If Goodwin system does not show any oscillation without decoy binding sites, a sustained oscillation is possible in their presence. The Goodwin system that oscillates without decoys remains oscillating in their presence. We also have observed that decoy presences can increase the amplitude and time period of the oscillation.

Finally, we have studied the precision the oscillation using stochastic simulations by quantifying the noise in time period and the amplitude. We have observed that the oscillation time period is more precise compared to the amplitudes. The presence of decoy can buffers the noise in the time period to a sub-Poissonian limit and the noise in the amplitude towards the Poissonian limit. Noise in gene expression has many advantageous and disadvantageous implications, from the phenotypic diversity, cell fate, to defects in developing embryos. Our results show the presence of decoys, depending on contexts, can enhance or buffer the noise as in an openloop circuit. However, it always reduces the noise in the oscillation where precision is crucial.

\section{ACKNOWLEDGMENT}

SD would like to thank M. Soltani and D. Sengupta for useful discussions. This work is supported by the National Science Foundation Grant ECCS-1711548.

\section{REFERENCES}

[1] M. B. Elowitz, A. J. Levine, E. D. Siggia, and P. S. Swain, "Stochastic gene expression in a single cell," Science, vol. 297, pp. 1183-1186, 2002.

[2] E. M. Ozbudak, M. Thattai, I. Kurtser, A. D. Grossman, and A. van Oudenaarden, "Regulation of noise in the expression of a single gene," Nature Genetics, vol. 31, pp. 69-73, 2002.

[3] W. J. Blake, M. Kaern, C. R. Cantor, and J. J. Collins, "Noise in eukaryotic gene expression," Nature, vol. 422, pp. 633-637, 2003.

[4] A. Eldar and M. B. Elowitz, "Functional roles for noise in genetic circuits," Nature, vol. 467, pp. 167-173, 2010.

[5] B. Munsky, G. Neuert, and A. van Oudenaarden, "Using gene expression noise to understand gene regulation," Science, vol. 336, pp. 183-187, 2012.

[6] J. M. Raser and E. K. O'Shea, "Noise in gene expression: Origins, consequences, and control," Science, vol. 309, pp. 2010 - 2013, 2005.

[7] A. Raj and A. van Oudenaarden, "Nature, nurture, or chance: stochastic gene expression and its consequences," Cell, vol. 135, pp. 216-226, 2008.

[8] R. Losick and C. Desplan, "Stochasticity and cell fate," Science, vol. 320, pp. 65-68, 2008.

[9] C. J. Davidson and M. G. Surette, "Individuality in bacteria," Annual Review of Genetics, vol. 42, pp. 253-268, 2008.

[10] D. Fraser and M. Kaern, "A chance at survival: gene expression noise and phenotypic diversification strategies," Mol Microbiol, vol. 71, pp. 1333-1340, 2009.
[11] S. M. Shaffer, M. C. Dunagin, S. R. Torborg, E. A. Torre, B. Emert, C. Krepler, M. Beqiri, K. Sproesser, P. A. Brafford, M. Xiao, E. Eggan, I. N. Anastopoulos, C. A. Vargas-Garcia, A. Singh, K. L. Nathanson, M. Herlyn, and A. Raj, "Rare cell variability and drug-induced reprogramming as a mode of cancer drug resistance," Nature, vol. 546, pp. 431-435, 2017

[12] A. Singh and L. S. Weinberger, "Stochastic gene expression as a molecular switch for viral latency," Current Opinion in Microbiology, vol. 12, pp. 460-466, 2009.

[13] J. Lewis, "Autoinhibition with transcriptional delay: A simple mechanism for the zebrafish somitogenesis oscillator," Current Biology, vol. 13, no. 16, pp. 1398 - 1408, 2003.

[14] S. Keskin, G. S. Devakanmalai, S. B. Kwon, H. Vu, M. Soltani, A. Singh, A. Ay, and E. M. Ozbudak, "Noise in the vertebrate segmentation clock is boosted by time-delays but tamed by notch signaling," Cell Reports, vol. 23, pp. 2175-2185, 2018.

[15] S. S. Golden, M. Ishiura, C. H. Johnson, and T. Kondo, "Cyanobacterial circadian rhythms," Annual Review of Plant Physiology and Plant Molecular Biology, vol. 48, no. 1, pp. 327-354, 1997.

[16] V. Dvornyk, O. Vinogradova, and E. Nevo, "Origin and evolution of circadian clock genes in prokaryotes," Proceedings of the National Academy of Sciences, vol. 100, no. 5, pp. 2495-2500, 2003.

[17] D. E. Nelson, A. E. C. Ihekwaba, M. Elliott, J. R. Johnson, C. A. Gibney, B. E. Foreman, G. Nelson, V. See, C. A. Horton, D. G. Spiller, S. W. Edwards, H. P. McDowell, J. F. Unitt, E. Sullivan, R. Grimley, N. Benson, D. Broomhead, D. B. Kell, and M. R. H. White, "Oscillations in nf-kb signaling control the dynamics of gene expression,” Science, vol. 306, no. 5696, pp. 704-708, 2004.

[18] B. Novák and J. J. Tyson, "Design principles of biochemical oscillators," Nature Reviews Molecular Cell Biology, vol. 9, p. 981, 2008.

[19] B. C. Goodwin, "Oscillatory behavior in enzymatic control processes," Advances in Enzyme Regulation, vol. 3, pp. 425-437, 1965.

[20] C. A. Kemme, D. Nguyen, A. Chattopadhyay, and J. Iwahara, "Regulation of transcription factors via natural decoys in genomic dna," Transcription, vol. 7, no. 4, pp. 115-120, 2016.

[21] R. Morishita, G. H. Gibbons, M. Horiuchi, K. E. Ellison, M. Nakama, L. Zhang, Y. Kaneda, T. Ogihara, and V. J. Dzau, "A gene therapy strategy using a transcription factor decoy of the e2f binding site inhibits smooth muscle proliferation in vivo," Proceedings of the National Academy of Sciences, vol. 92, no. 13, pp. 5855-5859, 1995.

[22] M. J. MANN, "Transcription factor decoys: A new model for disease intervention," Annals of the New York Academy of Sciences, vol. 1058, no. 1, pp. 128-139, 2005.

[23] M. Hecker and A. H. Wagner, "Transcription factor decoy technology: A therapeutic update," Biochemical Pharmacology, vol. 144, pp. $29-$ 34, 2017.

[24] M. Francois, P. Donovan, and F. Fontaine, "Modulating transcription factor activity: Interfering with protein-protein interaction networks," Seminars in Cell and Developmental Biology, 2018.

[25] T. Lee and N. Maheshri, "A regulatory role for repeated decoy transcription factor binding sites in target gene expression," Molecular systems biology, vol. 8, p. 576, 2012.

[26] A. Burger, A. M. Walczak, and P. G. Wolynes, "Abduction and asylum in the lives of transcription factors," Proceedings of the National Academy of Sciences, vol. 107, pp. 4016-4021, 2010.

[27] — , "Influence of decoys on the noise and dynamics of gene expression," Phes. Rev. E, vol. 86, p. 041920, 2012.

[28] M. Soltani, P. Bokes, Z. Fox, and A. Singh, "Nonspecific transcription factor binding can reduce noise in the expression of downstream proteins," Physical Biology, vol. 12, p. 055002, 2015.

[29] D. Das, S. Dey, R. C. Brewster, and S. Choube, "Effect of transcription factor resource sharing on gene expression noise," PLoS Comput Biol, vol. 13, p. e1005491, 2017.

[30] S. Dey and A. Singh, "Stochastic analysis of feedback control by molecular sequestration," in 2019 American Control Conference (ACC), 2019, pp. 4466-4471.

[31] Z. Wang, D. A. Potoyan, and P. G. Wolynes, "Molecular stripping, targets and decoys as modulators of oscillations in the nf-kb/ikba/dna genetic network," J R Soc Interface, vol. 13, no. 122, p. 20160606 , 2016.

[32] S. Jayanthi and D. Del Vecchio, "Tuning genetic clocks employing dna binding sites," PLOS ONE, vol. 7, no. 7, pp. 1-10, 072012.

[33] D. Thomas and M. Tyers, "Transcriptional regulation: Kamikaze activators," Current Biology, vol. 10, no. 9, pp. R341 - R343, 2000. 
[34] N. Van Kampen, Stochastic Processes in Physics and Chemistry. Elsevier, 2011

[35] D. T. Gillespie, "A general method for numerically simulating the stochastic time evolution of coupled chemical reactions," Journal of Computational Physics, vol. 22, pp. 403-434, 1976.

[36] J. Paulsson, "Model of stochastic gene expression," Physics of Life Reviews, vol. 2, pp. 157-175, 2005.

[37] V. Shahrezaei and P. S. Swain, "Analytical distributions for stochastic gene expression," Proceedings of the National Academy of Sciences, vol. 105, pp. 17 256-17261, 2008.

[38] V. Elgart, T. Jia, A. T. Fenley, and R. Kulkarni, "Connecting protein and mrna burst distributions for stochastic models of gene expression," Physical biology, vol. 8, p. 046001, 2011.

[39] A. Singh and P. Bokes, "Consequences of mRNA transport on stochastic variability in protein levels," Biophysical Journal, vol. 103, pp. 1087-1096, 2012.

[40] D. J. Wilkinson, Stochastic Modelling for Systems Biology. Chapman and Hall/CRC, 2011.

[41] D. A. McQuarrie, "Stochastic approach to chemical kinetics," Journal of Applied Probability, vol. 4, pp. 413-478, 1967.

[42] B. Munsky, W. S. Hlavacek, and L. S. Tsimring, Quantitative biology : theory, computational methods, and models. The MIT Press, 2018.

[43] S. Modi, M. Soltani, and A. Singh, "Linear noise approximation for a class of piecewise deterministic markov processes," in 2018 Annual American Control Conference (ACC), 2018, pp. 1993-1998.

[44] J. Elf and M. Ehrenberg, "Fast evaluation of fluctuations in biochemical networks with the linear noise approximation," Genome Research, vol. 13, pp. 2475-2484, 2003.

[45] P. Thomas, A. V. Straube, and R. Grima, "The slow-scale linear noise approximation: an accurate, reduced stochastic description of biochemical networks under timescale separation conditions," $B M C$ Systems Biology, vol. 6, p. 39, 2012.
[46] A. Singh and J. P. Hespanha, "Approximate moment dynamics for chemically reacting systems," IEEE Transactions on Automatic Control, vol. 56, pp. 414-418, 2011.

[47] C. S. Gillespie, "Moment closure approximations for mass-action models," IET Systems Biology, vol. 3, pp. 52-58, 2009.

[48] A. Singh and J. P. Hespanha, "Stochastic hybrid systems for studying biochemical processes," Philosophical Transactions of the Royal Society A, vol. 368, pp. 4995-5011, 2010.

[49] M. Soltani, C. Vargas, and A. Singh, "Conditional moment closure schemes for studying stochastic dynamics of genetic circuits," IEEE Transactions on Biomedical Systems and Circuits, vol. 9, pp. 518-526, 2015.

[50] D. Schnoerr, G. Sanguinetti, and R. Grima, "Comparison of different moment-closure approximations for stochastic chemical kinetics," The Journal of Chemical Physics, vol. 143, p. 185101, 2015.

[51] E. Lakatos, A. Ale, P. D. W. Kirk, and M. P. H. Stumpf, "Multivariate moment closure techniques for stochastic kinetic models," The Journal of Chemical Physics, vol. 143, p. 094107, 2015.

[52] P. Smadbeck and Y. N. Kaznessis, "A closure scheme for chemical master equations," Proceedings of the National Academy of Sciences, vol. 110, pp. 14261-14 265, 2013.

[53] M. Thattai and A. van Oudenaarden, "Intrinsic noise in gene regulatory networks," Proceedings of the National Academy of Sciences, vol. 98 , pp. 8614-8619, 2001.

[54] R. Séroul, Polynomials. Berlin, Heidelberg: Springer Berlin Heidelberg, 2000, pp. 253-295.

[55] J. Griffith, "Mathematics of cellular control processes i. negative feedback to one gene," Journal of Theoretical Biology, vol. 20, no. 2 , pp. $202-208,1968$. 